University and Bell Labs, Lucent Technologies, as a visiting scholar. $\mathrm{He}$ is also a chief scientist and a team leader at RIKEN, Japan.

Someya's current research interests include organic transistors, flexible electronics, plastic integrated circuits, large-area sensors, and plastic actuators. He has received a number of awards, including the Japan Society for the Promotion of Science Prize, the 2008 German Innovation Award 1st Prize, the 2004 IEEE/ISSCC Sugano Award, and the 2009 IEEE Paul Rappaport Award. He was a global scholar at Princeton University, a member of the MRS Board of Directors (2009-2011), and an IEEE/EDS Distinguished Lecturer (2005-2016). His "large-area sensor array" electronic thin film was featured in Time Magazine as one of the "Best Inventions of 2005."

\title{
75th Device Research Conference to be held June 25-28
}

www.deviceresearchconference.org

$\mathrm{T}^{\mathrm{h}}$ he 75th Device Research Conference (DRC) will be held June 25-28, 2017, at the University of Notre Dame, Indiana, USA, and is co-sponsored by the Materials Research Society and the IEEE Electron Devices Society. The general chair is Debdeep Jena of Cornell University, technical program chair is Aaron Franklin of Duke University, and technical program vice chair is Deji Akinwande of The University of Texas at Austin.
The DRC brings together scientists, researchers, and students from around the world to share their latest discoveries in device science, technology, and modeling. Topics include but are not limited to: biological devices, emerging electronic device materials, energy scavenging devices, magnetic devices, memory devices, and modeling and simulation of devices. Plenary speakers are Tsu-Jae King Liu of the University of California, Berkeley;
Hideo Hosono of the Tokyo Institute of Technology; and John Bowers of the University of California, Santa Barbara. There will also be invited speakers, short courses, and evening rump sessions. Technical sessions will be accented by special events highlighting the anniversary of the DRC.

More information can be accessed from the conference website at www. deviceresearchconference.org.

\section{7th International Conference on Hard X-Ray Photoelectron Spectroscopy to be held} September 11-15 https://sites.google.com/a/lbl.gov/haxpes2017

$\mathrm{T}$ he 7th International Conference on Hard X-Ray Photoelectron Spectroscopy (HAXPES) will be held September 11-15, 2017, in Berkeley, Calif., USA. Co-chairs are Charles Fadley of the University of California, Davis, and Lawrence Berkeley National Laboratory, USA; Zahid Hussain of Lawrence Berkeley National Laboratory, USA; and Piero A. Pianetta of Stanford University/SLAC
National Accelerator Laboratory, USA.

HAXPES brings together researchers from a variety of fields, from fundamental condensed matter and atomic and molecular physics to more applied surface and interface studies of catalysis, energy and IT device- and process development, and environmental research. HAXPES involves photon energies in the multi-keV range above $\sim 2 \mathrm{keV}$, but the conference also encourages presentations involving complementary photoemission measurements at lower energies, as well as other $\mathrm{x}$-ray-based techniques.

The abstract submission deadline is June 15. More information can be accessed from the conference website at https://sites.google.com/a/lbl.gov/ haxpes2017 or by email at haxpes2017@ gmail.com.
Text Generation Electrochemistry 1 (NGenE) will be held June 26-30, 2017, in Illinois, USA. The 2017 program will explore the theme: "Phenomena at Electrochemical Interfaces" and will consist of a combination of lectures and discussions, research project planning and mentoring, and demonstrations. The participants will consist of
25 advanced graduate students and 10 distinguished lecturers.

Faculty will present a series of critical questions, while motivating their importance through past experiences and future societal needs. They will also highlight the need for an accurate definition of chemical components at heterogeneous interfaces, down to the atomic level and under operating conditions. Emerging techniques to probe and model interfacial phenomena will be presented and discussed. The students will assemble into teams charged with one of the challenges and will develop a scientific program to solve the challenge using innovative experiments and theory.

For more information, visit https:// energyinitiative.uic.edu/energy/ngene. 